\title{
A matched cohort comparison of clinical outcomes following microsurgical resection or stereotactic radiosurgery for patients with small- and medium-sized vestibular schwannomas
}

\author{
John G. Golfinos, MD, ${ }^{1}$ Travis C. Hill, PhD, ${ }^{1}$ Rae Rokosh, BS, ${ }^{1}$ Osamah Choudhry, MD, ${ }^{1}$ \\ Matthew Shinseki, BS, ${ }^{1}$ Alireza Mansouri, MD, ${ }^{1}$ David R. Friedmann, MD, ${ }^{2}$ \\ J. Thomas Roland Jr., MD, ${ }^{2}$ and Douglas Kondziolka, MD' \\ Departments of ${ }^{1}$ Neurosurgery and ${ }^{2}$ Otolaryngology, NYU Langone Medical Center, New York University, New York, New York
}

OBJECTIVE A randomized trial that compares clinical outcomes following microsurgery (MS) or stereotactic radiosurgery (SRS) for patients with small- and medium-sized vestibular schwannomas (VSs) is impractical, but would have important implications for clinical decision making. A matched cohort analysis was conducted to evaluate clinical outcomes in patients treated with MS or SRS.

METHODS The records of 399 VS patients who were cared for by 2 neurosurgeons and 1 neurotologist between 2001 and 2014 were evaluated. From this data set, 3 retrospective matched cohorts were created to compare hearing preservation (21 matched pairs), facial nerve preservation ( 83 matched pairs), intervention-free survival, and complication rates (85 matched pairs) between cases managed with SRS and patients managed with MS. Cases were matched for age at surgery ( \pm 10 years) and lesion size $( \pm 0.1 \mathrm{~cm})$. To compare hearing outcomes, cases were additionally matched for preoperative Class A hearing according to the American Academy of Otolaryngology-Head and Neck Surgery guidelines. To compare facial nerve (i.e., cranial nerve [CN] VII) outcomes, cases were additionally matched for preoperative House-Brackmann (HB) score. Investigators who were not involved with patient care reviewed the clinical and imaging records. The reported outcomes were as assessed at the time of the last follow-up, unless otherwise stated.

RESULTS The preservation of preoperative Class A hearing status was achieved in $14.3 \%$ of MS cases compared with $42.9 \%$ of SRS cases (OR $4.5 ; p<0.05$ ) after an average follow-up interval of 43.7 months and 30.3 months, respectively. Serviceable hearing was preserved in $42.8 \%$ of MS cases compared with $85.7 \%$ of SRS cases (OR $8.0 ; p<0.01$ ). The rates of postoperative $\mathrm{CN}$ VII dysfunction were low for both groups, although significantly higher in the MS group (HB III-IV $11 \%$ vs $0 \%$ for SRS; OR 21.3; $p<0.01$ ) at a median follow-up interval of 35.7 and 19.0 months for MS and SRS, respectively. There was no difference in the need for subsequent intervention (2 MS patients and 2 SRS patients).

CONCLUSIONS At this high-volume center, VS resection or radiosurgery for tumors $\leq 2.8 \mathrm{~cm}$ in diameter was associated with low overall morbidity. The need for subsequent intervention was the same in both groups. SRS was associated with improved hearing and facial preservation rates and reduced morbidity, but with a shorter average follow-up period. Facial function was excellent in both groups. Since patients were not randomly selected for surgery, different clinical outcomes may be of different value to individual patients. Both anticipated medical outcomes and patient goals remain the drivers of treatment decisions.

http://thejns.org/doi/abs/10.3171/2015.12.JNS151857

KEY WORDS vestibular schwannoma; Gamma Knife; stereotactic radiosurgery; microsurgery; facial nerve; hearing preservation 
$\mathrm{V}$ ESTIBULAR schwannomas (VSs) are benign tumors that arise from the vestibular division of the eighth cranial nerve. ${ }^{16}$ VSs represent approximately 5\% of all intracranial tumors and are commonly diagnosed in the fifth and sixth decades of life. ${ }^{27}$ The incidence of VS has been estimated to vary from approximately 1 to 2 per 100,000 person-years in recent studies. ${ }^{16,18,24}$ The growth rate of VS is estimated to fall in the range of 0.1 to $0.3 \mathrm{~cm} /$ year ${ }^{11}$ with a volumetric tumor doubling time of 1.7 to 4.4 years. ${ }^{26}$ At the initial diagnosis, patients most often present with unilateral high-frequency sensorineural hearing loss, followed by imbalance, tinnitus, and headaches, ${ }^{22}$ these symptoms of brainstem compression and obstructive hydrocephalus may be associated with lesions larger than $3 \mathrm{~cm} .{ }^{10}$ The management options for VS include surveillance with serial imaging or treatment with either microsurgery (MS) or radiosurgery (SRS).

When SRS first became available, MS was the treatment modality of choice for VS, and SRS was reserved for cases in which surgery was medically contraindicated. However, SRS gained recognition in numerous reports as a viable treatment option that offers good long-term tumor growth control with a favorable risk profile, particularly for lesions smaller than $3 \mathrm{~cm}$. Aside from hearing loss and facial nerve dysfunction, complications unique to MS have included postoperative headache (up to 18\%) and CSF leakage (up to $10 \%$ ). This increased morbidity has historically been balanced by the superior long-term tumor control and curative nature of MS. However, recent studies examining small- and medium-sized tumors have reported SRS growth control rates approaching $98 \%$ coupled with excellent preservation of hearing status within the first few years after SRS (60\%-90\% of patients), as well as preservation of facial nerve function (95\%-100\% of patients) and low overall procedural morbidity., ${ }^{3,16,31}$ As a result, SRS has increasingly been recommended as an equivalent therapeutic modality for small- and medium-sized VS.

Despite this equipoise, there has not been a randomized controlled trial that directly compares the safety and efficacy of SRS and MS for unilateral VS. ${ }^{21}$ At present, such a study is unlikely due to the strong role of patient choice and because it would require a long follow-up period given the slow growth rate of VS. Instead, the best available evidence relies on retrospective reviews and prospective series. A recent systematic review designed to assess the optimal intervention for small- to medium-sized VS-defined as 2.5 to $3.0 \mathrm{~cm}$ in maximal diameter-identified several reports that directly compared MS and SRS outcomes. ${ }^{23}$ All studies demonstrated favorable outcomes in patients managed with SRS, as reflected in the preservation of hearing status and facial nerve function. However, these studies are limited by their use of unmatched cohorts or low overall sample size.

In the absence of randomized controlled trials to address this question, well-designed observational studies that balance critical prognostic factors provide the most rigorous evidence. ${ }^{4}$ In the current retrospective institutional study, we sought to compare the long-term safety and efficacy of SRS and MS for the management of treatmentnaive unilateral VS not associated with neurofibromatosis Type II. To this end, we present an analysis of 2 large pa- tient cohorts that were matched for clinical demographics and baseline tumor characteristics.

\section{Methods \\ Patient Population}

This protocol was reviewed and approved by the New York University Institutional Review Board. We retrospectively reviewed the charts of all VS patients who were managed with either SRS or MS at our institution by 2 neurosurgeons and 1 neurotologist between 2001 and 2014. Treatment decisions were made with the patient after a thorough discussion of the risks and benefits of each management strategy. General treatment recommendations were made based on the natural history of disease, the neurological and medical status of the patient, and individual patient goals. Investigators who were not involved with patient care reviewed the clinical and imaging records. Study data were collected and managed using REDCap electronic data capture tools. ${ }^{12}$

After excluding confirmed neurofibromatosis Type II and bilateral VS cases, a total of 399 records were reviewed. We selected all 313 treatment-naive lesions with a tumor size $\leq 2.8 \mathrm{~cm}$ that were planned to undergo single-stage interventions (111 SRS and 202 MS lesions); of these, no cases were lost on follow-up. We then created matched cohorts that were tailored to each outcome (intervention-free survival, complications, and the preservation of hearing or facial nerve function). Individual case matching was performed with a custom algorithm written in MATLAB (MathWorks); SRS cases were matched with MS cases by age $( \pm 10$ years) and lesion size $( \pm 0.1 \mathrm{~cm})$. For analysis of hearing preservation, cases were additionally matched for preoperative hearing classification and filtered to exclude cases lacking postoperative audiograms. For the facial nerve dysfunction analysis, cases were additionally matched by preoperative House-Brackmann (HB) grade. Tumor size was estimated as the square root of the product of the maximal transverse and anteroposterior diameters of the tumor. A $2.8-\mathrm{cm}$ cutoff value was chosen to reflect the majority of SRS cases; tumors with larger diameters were not considered for SRS unless specific clinical criteria were met. The surgical approaches employed in this study included translabyrinthine, retrosigmoid, and middle cranial fossa craniotomies; translabyrinthine approaches were excluded from the hearing preservation analysis. The decision to perform the retrosigmoid approach was based on both clinical and anatomical factors and not always attempted for hearing preservation. ${ }^{14}$ All SRS cases were treated with a prescription dose $(50 \%$ of peak) of 12 to $13 \mathrm{~Gy}$.

\section{Hearing and Facial Symptoms}

Facial nerve function was assessed using the HB grading scale, and facial nerve dysfunction was rigorously defined as HB Grade III or greater, as noted in the clinical notes. In the assessment of hearing, audiometric data were reviewed and hearing was categorized according to the American Academy of Otolaryngology-Head and Neck Surgery (AAO-HNS) guidelines. Serviceable hearing was defined as AAO-HNS Class A or B. All reported facial 
nerve and hearing outcomes were as assessed at the last follow-up examination, unless otherwise stated. Intervention-free survival and complication rates were assessed by reviewing the chart notes and hospitalization records.

\section{Statistical Analysis}

Statistical analysis was performed using MATLAB, R, and Prism (GraphPad Software) software packages. Univariate analyses were performed with the log-rank test, Student t-test, Pearson's chi-square test, or Fisher's exact test, where appropriate.

\section{Results}

\section{Hearing Preservation}

In VS treatment, hearing preservation is a goal for some patients, and for certain small- and medium-sized tumors both SRS and hearing-preserving MS approaches may preserve serviceable hearing. To compare hearing preservation, 21 MS cases were matched with 21 SRS cases, including cases only treated with middle fossa and retrosigmoid approaches and cases only with Class A hearing preoperatively. For the SRS cohort, the chart records reported the mean and maximum cochlear dose for $13(61.9 \%)$ and 14 (66.7\%) patients, respectively. Of these cases, the median average cochlear dose was 3.3 Gy and the median maximum cochlear dose was 6.5 Gy (Table 1). Patients in the
MS and SRS cohorts were not significantly different in terms of any of the demographic characteristics assessed, although the follow-up interval showed a trend toward longer follow-up in the MS group (median 43.7 months vs 30.3 months, $\mathrm{p}>0.2$; Table 1). Within this follow-up period, the posttreatment preservation of serviceable hearing was greater after SRS than MS $(42.9 \%$ for MS vs $85.7 \%$ for SRS for Class A and B patients combined; OR 8.0; $p<$ 0.01 ; Fig. 1A) and the preservation of preoperative Class A hearing status was also better after SRS than MS (14.3\% for MS vs $42.9 \%$ for SRS; OR 4.5, p < 0.05; Fig. 1A). Within this SRS cohort, the median follow-up interval of the cases with preserved preoperative hearing status was 18.2 months compared with 32.1 months for cases with a decrement in hearing status $(p<0.05)$. The survival curves were significantly different between the MS and SRS cohorts in terms of the postoperative preservation of both serviceable hearing ( $\mathrm{p}<0.01$; Fig. 1B) and Class A hearing ( $\mathrm{p}<0.01$; Fig. 1C). Notably, the survival curves for the preservation of preoperative hearing status appear to converge by 60 months, but it should be considered that $66 \%$ (12 of 18) of the MS cases with the loss of preoperative hearing status were Class D, while $75 \%$ (9 of 12) of SRS cases with hearing loss remained Class B at the last follow-up examination. Overall, the loss of preoperative hearing status was delayed in the SRS cohort, whereas postoperative hearing status in the MS cohort was more often durable.

TABLE 1. Patient demographics of the matched cohorts for the hearing preservation analysis

\begin{tabular}{|c|c|c|c|}
\hline Variable & $\mathrm{MS}^{*}$ & SRS $^{*}$ & $p$ Value \\
\hline No. of patients & 21 & 21 & \\
\hline Median age, yrs & $50.6(36.7-64.6)$ & $54.1(41.1-69.4)$ & 0.32 \\
\hline Median lesion size, $\mathrm{cm}$ & $1.00(0.57-1.60)$ & $1.00(0.60-1.47)$ & 0.81 \\
\hline Median follow-up, mos & $43.7(4.8-115.7)$ & $30.3(10.0-90.8)$ & 0.21 \\
\hline Sex & & & 0.66 \\
\hline Male & $10(47.6)$ & $11(52.4)$ & \\
\hline Female & $11(52.4)$ & $10(47.6)$ & \\
\hline \multicolumn{4}{|c|}{ Symptoms prior to intervention } \\
\hline Tinnitus & $12(57.1)$ & $15(71.4)$ & 0.35 \\
\hline Vestibular dysfunction & $9(42.9)$ & $12(57.1)$ & 0.62 \\
\hline AAO-HNS hearing class & & & 1.00 \\
\hline A & $21(100.0)$ & $21(100.0)$ & \\
\hline $\mathrm{B}$ & $0(0.0)$ & $0(0.0)$ & \\
\hline Facial nerve dysfunction & & & 1.00 \\
\hline $\mathrm{HB}|\&| \mid$ & $21(100.0)$ & $21(100.0)$ & \\
\hline$H B \geq I I I$ & $0(0.0)$ & $0(0.0)$ & \\
\hline Koos grade & & & 0.13 \\
\hline 1 & $8(38.1)$ & $5(23.8)$ & \\
\hline 2 & $8(38.1)$ & $13(61.9)$ & \\
\hline 3 & $4(19.0)$ & $2(9.5)$ & \\
\hline 4 & $1(4.8)$ & $1(4.8)$ & \\
\hline \multicolumn{4}{|l|}{ SRS cochlear dose, Gy† } \\
\hline Median average dose & & $3.3(1.6-4.2)$ & \\
\hline Median maximum dose & & $6.5(3.4-8.4)$ & \\
\hline
\end{tabular}



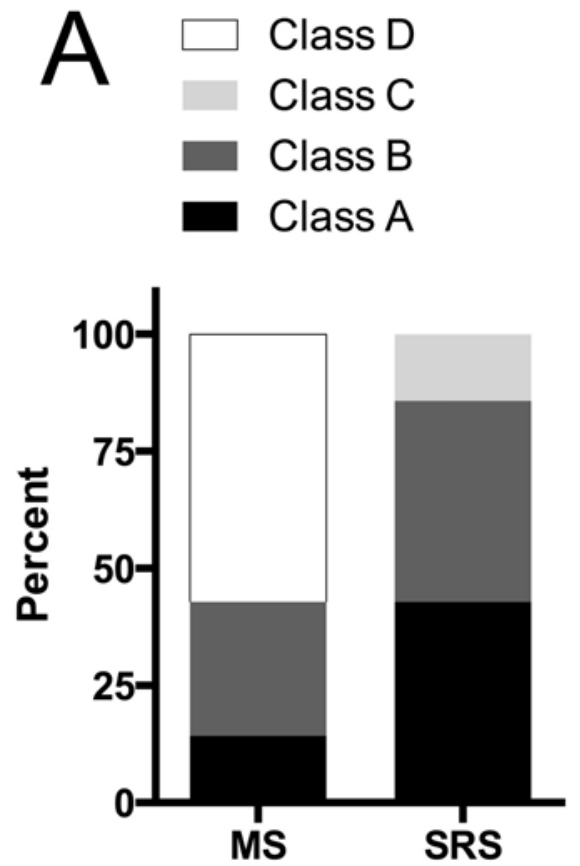

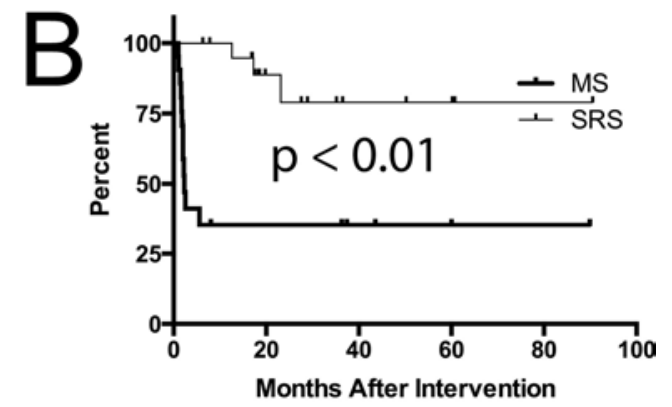

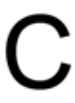

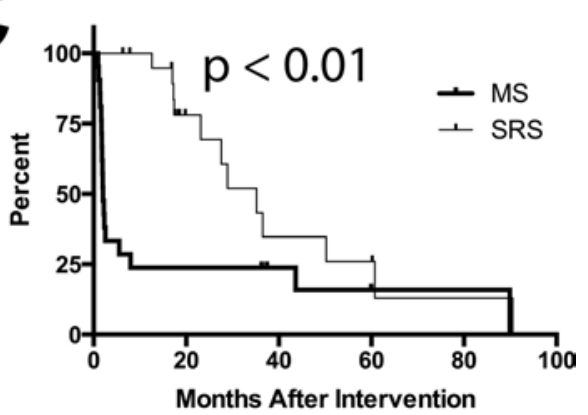

FIG. 1. SRS provides better hearing preservation than MS over short time scales. A: The percentage of all tumors $\leq 2.8 \mathrm{~cm}$ with hearing Class A-D after intervention in the MS and SRS cohorts $(n=21$ in each group). MS was associated with the reduced preservation of preoperative hearing status ( $14 \%$ vs $47 \% ; p<0.05)$ and serviceable hearing status $(43 \%$ vs $100 \% ; p<0.01)$ compared with SRS. B and C: Kaplan-Meier plots of serviceable hearing status preservation (B) and preoperative hearing status preservation $(C)$ in the MS and SRS cohorts both demonstrate significant differences in survivorship $(p<0.01)$.

\section{Facial Symptoms}

One of the greatest concerns related to quality of life after VS treatment is facial nerve dysfunction. For comparison of postoperative facial nerve dysfunction, $83 \mathrm{MS}$ cases with preoperative HB Grade I were matched with 83 SRS cases with preoperative HB Grade I. The MS cohort included a combination of translabyrinthine, retrosigmoid, and middle fossa craniotomies. Patients in the MS and SRS cohorts differed only in terms of the followup period (median 35.7 months vs 19 months; $\mathrm{p}<0.05$; Table 2). At the time of the last follow-up examination, the incidence of facial nerve dysfunction was higher after MS than SRS (11\% vs 0\%; OR 21.3; p < 0.01; Fig. 2, left). Within the MS cohort, 9 of 83 patients developed persistent facial nerve dysfunction after surgery. Within the SRS cohort, no patient developed facial nerve dysfunction (HB Grades III-VI) after radiosurgery that persisted at the last follow-up examination. The majority of cases in both the MS and SRS groups maintained their preoperative HB grade, although worse postoperative facial nerve function was more often seen after MS than SRS (30\% of MS vs $4 \%$ of SRS patients; OR 8.5; p < 0.01; Fig. 2, right).

\section{Intervention-Free Survival}

To compare intervention-free survival, 85 MS cases were matched with 85 SRS cases. The MS cohort included a mixture of translabyrinthine, retrosigmoid, and middle fossa approaches. Patients who underwent MS and SRS differed in terms of the median follow-up time (36.8 months vs 20 months; $p<0.01$ ) and mode of presentation (100\% symptomatic vs $91 \%$ symptomatic, $\mathrm{p}<0.01$; Table
3). Following the initial intervention, there was no difference in intervention-free survival for VS treated with MS or SRS (2 treatment failures in each cohort; $p=0.48$; Fig. $3)$. The characteristics of the cases that required subsequent intervention are presented in Table 4. Within the MS cohort, cases requiring subsequent intervention $(n=2)$ had longer follow-up duration (36.5 months vs 88.2 months), better preoperative hearing status (serviceable hearing rates of $100 \%$ vs $65 \%$ ), underwent treatment via the retrosigmoid approach ( 2 of 2 patients), and were more likely to have undergone non-total (near-total or subtotal) resection (50\% vs 2.4\%). In contrast, treatment failures in the SRS cohort were not significantly different from the successes along any of the metrics assessed. Overall, failure rates were low, which reduced the sensitivity of our analysis; given this limitation, we conclude that MS and SRS do not differ in terms of the rate of subsequent intervention for VS presenting with a maximal diameter $\leq 2.8 \mathrm{~cm}$.

\section{Tumor Control}

In the present study, the need for subsequent intervention was the primary tumor-control endpoint. However, we chose to examine radiographic tumor control to assess both the extent of radiographic tumor control in 1) MS cases with recurrence or non-total resection and 2) SRS cases. Of the $85 \mathrm{MS}$-managed cases, 3 were excluded from this analysis due to the lack of documented lesion size after intervention. Seventy-five of $82(91.5 \%)$ lesions underwent total resection with no detectable residual tumor or recurrence within the study period. Two of the remaining 82 lesions (2.4\%) in the MS cohort required salvage 
TABLE 2. Patient demographics of the matched cohort for the facial nerve dysfunction analysis

\begin{tabular}{lccc}
\hline \multicolumn{1}{c}{ Variable } & \multicolumn{1}{c}{ MS $^{*}$} & \multicolumn{1}{c}{ SRS } & p Value \\
\hline No. of patients & 83 & 83 & 0.31 \\
\hline Median age, yrs & $57.9(29.5-72.5)$ & $58.7(32.1-75.1)$ & 0.78 \\
\hline Median lesion size, cm & $1.22(0.40-2.42)$ & $1.20(0.40-2.50)$ & 0.02 \\
\hline Median follow-up, mos & $35.7(0.3-115.7)$ & $19.0(2.4-90.8)$ & 0.27 \\
\hline Sex & & & \\
\hline Male & $46(55.4)$ & $41(49.4)$ & 0.35 \\
\hline Female & $37(44.6)$ & $42(50.6)$ & 0.35 \\
\hline Symptoms prior to intervention & & & 1.00 \\
\hline Tinnitus & $43(51.8)$ & $49(59.0)$ & \\
\hline Vestibular dysfunction & $42(50.6)$ & $50(60.2)$ & \\
\hline Facial nerve dysfunction & & $83(100.0)$ & 0.26 \\
\hline HB I & $83(100.0)$ & $0(0.0)$ & \\
\hline HB $\geq l$ & $0(0.0)$ & $15(18.1)$ & \\
\hline Koos grade & $19(22.9)$ & $39(47.0)$ & \\
\hline 1 & $32(38.6)$ & $20(24.1)$ & \\
\hline 3 & $7(8.4)$ & $25(30.1)$ & \\
\hline 4 & & \\
\hline
\end{tabular}

* Values are shown as the median (range) or count (\%).

therapy after 52.1 and 90.7 months, respectively. These 2 lesions initially underwent non-total resection and were subsequently managed with SRS. One additional lesion in the MS management group is currently under annual surveillance due to radiographic tumor progression after 39.3 months of follow-up following total resection. The remaining 4 lesions -1 subtotal and 3 total resectionswere stable at the last follow-up examination (range 6-58 months) (Fig. 4A).

Of the 85 SRS managed cases, 2 cases were excluded from radiographic analysis due to the lack of a documented lesion size after intervention. The majority of the le- sions in the SRS cohort demonstrated little fluctuation in size after therapy, but those lesions that demonstrated a sustained increase in size tended toward failure (Fig. 4C and D). Two of 83 lesions (2.4\%) in the SRS cohort failed initial treatment and required salvage therapy after 31.4 and 50.6 months, respectively. The reasons for failure were radiographic progression in 1 case, and symptomatic progression including recurrence of dizziness, ear fullness, and worsened tinnitus in the second case. All cases in the SRS cohort in which initial treatment failed subsequently underwent salvage MS. The remaining 4 lesions that demonstrated $\geq 1.5$-fold change in size after SRS management
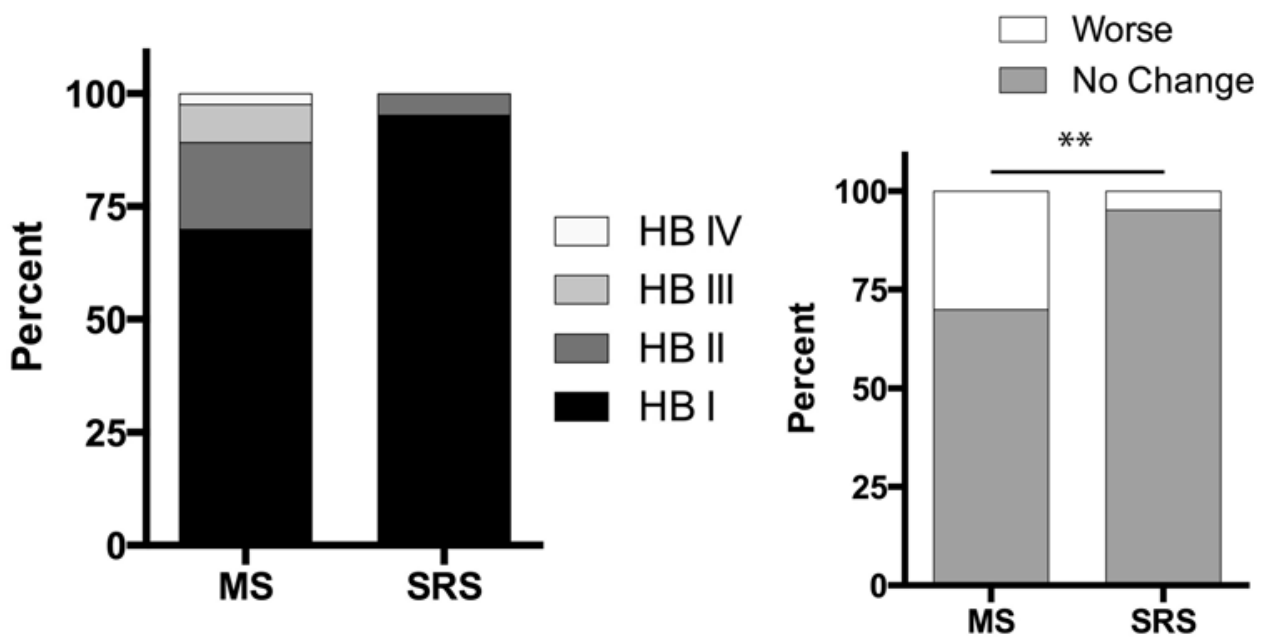

FIG. 2. SRS provides better overall preservation of facial nerve function. Left: Percentage of all tumors $\leq 2.8 \mathrm{~cm}$ by HB grade after surgery in the MS and SRS groups ( $n=83$ in each group). MS was associated with a greater rate of postoperative facial nerve dysfunction than SRS $(10.8 \% \mathrm{HB}$ Grade III-VI vs $0 \% \mathrm{HB}$ Grade III-VI; $p<0.01)$. Right: Relative to preoperative HB grade, MS cases were more likely than SRS cases to demonstrate a worse HB grade $\left(30 \%\right.$ vs $4 \%$, respectively; $\left.{ }^{* *} p<0.01\right)$. 
TABLE 3. Patient demographics of the matched cohort for tumor control and complications analysis

\begin{tabular}{|c|c|c|c|}
\hline Variable & $\mathrm{MS}^{*}$ & $\mathrm{SRS}^{*}$ & $p$ Value \\
\hline No. of patients & 85 & 85 & \\
\hline Age, yrs & $58.0(27.9-71.6)$ & $59.0(32.1-75.1)$ & 0.40 \\
\hline Lesion size, $\mathrm{cm}$ & $1.22(0.55-2.45)$ & $1.20(0.40-2.50)$ & 0.76 \\
\hline Follow-up interval, mos & $36.8(1.4-115.7)$ & $20.0(2.4-90.8)$ & $<0.01$ \\
\hline Sex & & & 0.66 \\
\hline Male & $39(45.9)$ & $41(48.2)$ & \\
\hline Female & $46(54.1)$ & $44(51.8)$ & \\
\hline Lesion side & & & 0.19 \\
\hline Right & $46(54.1)$ & $40(47.1)$ & \\
\hline Left & $39(45.9)$ & $45(52.9)$ & \\
\hline Presentation & & & $<0.01$ \\
\hline Incidental & $0(0.0)$ & $8(9.4)$ & \\
\hline Symptomatic & $85(100.0)$ & $77(90.6)$ & \\
\hline \multicolumn{4}{|c|}{ Symptoms prior to intervention } \\
\hline Tinnitus & $42(49.4)$ & $50(58.8)$ & 0.22 \\
\hline Vestibular dysfunction & $46(54.1)$ & $52(61.2)$ & 0.79 \\
\hline Hearing status & & & 0.62 \\
\hline Serviceable & $56(65.9)$ & $57(67.1)$ & \\
\hline Not serviceable & $23(27.1)$ & $20(23.5)$ & \\
\hline No audiogram & $6(7.1)$ & $8(9.4)$ & \\
\hline Facial nerve dysfunction & & & 1.00 \\
\hline HB Grade I/II & $85(100.0)$ & $85(100.0)$ & \\
\hline HB Grade $\geq$ III & $0(0.0)$ & $0(0.0)$ & \\
\hline Koos grade & & & 0.29 \\
\hline 1 & $18(21.2)$ & $17(20.0)$ & \\
\hline 2 & $32(37.6)$ & $38(44.7)$ & \\
\hline 3 & $7(8.2)$ & $9(10.6)$ & \\
\hline 4 & $28(32.9)$ & $21(24.7)$ & \\
\hline
\end{tabular}

* Values are shown as the median (range) or count (\%).

were symptomatically and radiographically stable at the last follow-up examination (range 5-65 months) (Fig. 4B).

\section{Complications}

The complication rates for both treatment modalities were low overall (Table 5). The most frequently reported morbidities within the MS group included headache $(16.5 \%)$, change in taste $(5.9 \%)$, CSF leakage (4.7\%), fatigue (3.5\%), infection (2.4\%), and hydrocephalus, deep venous thrombosis (DVT), lower cranial nerve dysfunction, and seroma (1.4\% each). Most cases of headache occurred during the early postoperative period and were resolved within 2 months; only 4 cases (4.7\%) presented with persistent headache at 9,23,38, and 100 months, respectively. Likewise, all but 2 cases of gustatory changes resolved within 2 months, and only 2 cases (2.4\%) presented with persistent changes in taste at 19 and 49 months. All cases of CSF leakage resolved, and none required further intervention. One case of infection responded to antibiotics and did not require intervention, while the second case required debridement and bone flap removal without longterm disability. The only instance of DVT was managed

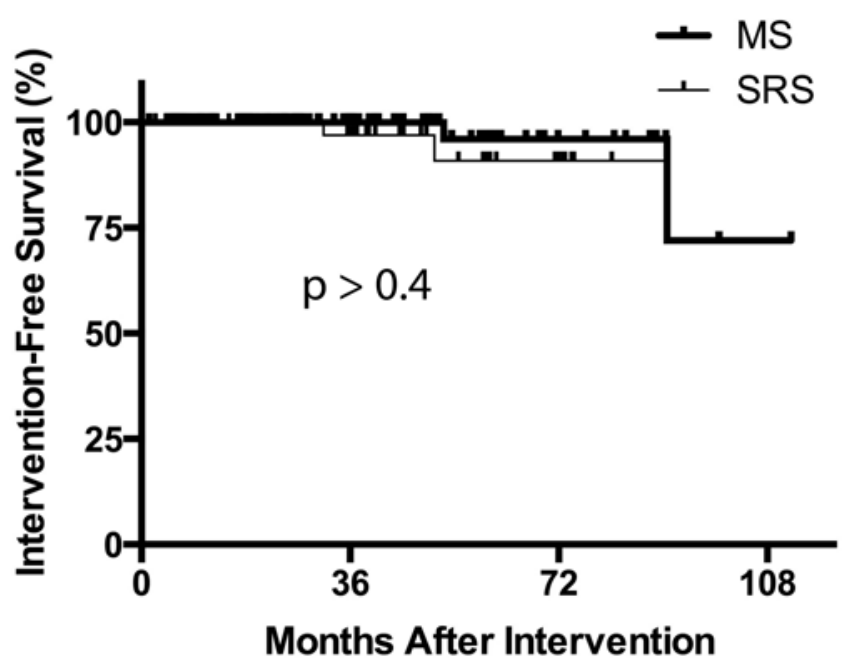

FIG. 3. Kaplan-Meier plot of intervention-free survival in the MS and SRS groups ( $p>0.4 ; n=85$ for each group). 
TABLE 4. Characteristics of cases requiring subsequent intervention

\begin{tabular}{|c|c|c|c|c|}
\hline \multirow[b]{2}{*}{ Variable } & \multicolumn{2}{|c|}{$\mathrm{MS}^{*}$} & \multicolumn{2}{|c|}{ SRS $^{*}$} \\
\hline & Success & Failure & Success & Failure \\
\hline No. of patients & 83 & 2 & 83 & 2 \\
\hline Median age, yrs & $58.2(27.9-71.6)$ & $53.0(50.4-55.5)$ & $59.0(32.1-75.1)$ & $60.0(56.4-63.5)$ \\
\hline Median lesion size, $\mathrm{cm}$ & $1.22(0.55-2.45)$ & $1.35(1.20-1.50)$ & $1.20(0.40-2.50)$ & $1.26(1.02-1.50)$ \\
\hline Median follow-up, mos & $36.5(1.4-112.1)$ & $88.2(60.7-115.7)$ & $19.0(2.4-90.8)$ & $42.1(32.8-51.4)$ \\
\hline \multicolumn{5}{|l|}{ Sex } \\
\hline Male & $38(45.8)$ & $1(50.0)$ & $41(49.4)$ & $0(0.0)$ \\
\hline Female & $45(54.2)$ & $1(50.0)$ & $42(50.6)$ & $2(100.0)$ \\
\hline \multicolumn{5}{|l|}{ Lesion side } \\
\hline Right & $44(53.0)$ & $2(100.0)$ & $40(48.2)$ & $0(0.0)$ \\
\hline Left & $39(47.0)$ & $0(0.0)$ & $43(51.8)$ & $2(100.0)$ \\
\hline \multicolumn{5}{|l|}{ Mode of presentation } \\
\hline Incidental & $0(0.0)$ & $0(0.0)$ & $8(9.6)$ & $0(0.0)$ \\
\hline Symptomatic & $83(100.0)$ & $2(100.0)$ & $75(90.4)$ & $2(100.0)$ \\
\hline \multicolumn{5}{|l|}{ Hearing status } \\
\hline Serviceable & $54(65.1)$ & $2(100.0)$ & $56(67.5)$ & $1(50.0)$ \\
\hline Not serviceable & $23(27.7)$ & $0(0.0)$ & $19(22.9)$ & $1(50.0)$ \\
\hline No audiogram & $6(7.2)$ & $0(0.0)$ & $8(9.6)$ & $0(0.0)$ \\
\hline \multicolumn{5}{|l|}{$\mathrm{HB}$} \\
\hline 1 & $80(96.4)$ & $2(100.0)$ & $82(98.8)$ & $2(100.0)$ \\
\hline II & $3(3.6)$ & $0(0.0)$ & $1(1.2)$ & $0(0.0)$ \\
\hline \multicolumn{5}{|l|}{ Koos grade } \\
\hline 1 & $18(21.7)$ & $0(0.0)$ & $17(20.5)$ & $0(0.0)$ \\
\hline 2 & 31 (37.3) & $1(50.0)$ & $36(43.4)$ & $2(100.0)$ \\
\hline 3 & $6(7.2)$ & $1(50.0)$ & $9(10.8)$ & $0(0.0)$ \\
\hline 4 & $28(33.7)$ & $0(0.0)$ & $21(25.3)$ & $0(0.0)$ \\
\hline \multicolumn{5}{|l|}{ Surgical approach } \\
\hline Retrosigmoid & 34 & 2 & & \\
\hline Translabyrinthine & 40 & 0 & & \\
\hline Middle fossa & 9 & 0 & & \\
\hline \multicolumn{5}{|l|}{ Extent of resection } \\
\hline Total & 81 & 1 & & \\
\hline Subtotal & 2 & 1 & & \\
\hline
\end{tabular}

* Values are shown as the median (range) or count (\%).

medically without sequelae. The only case of lower cranial nerve dysfunction consisted of intermittent hoarseness and dysphagia that was not clearly related to surgery. The patient's symptoms improved without intervention and were subjectively characterized as only minimally bothersome at the last follow-up examination. The only case of postoperative hydrocephalus required hospitalization and was treated with 2 large-volume spinal taps, but the patient recovered fully with no long-term disability. After SRS, morbidities included headache $(5.9 \%)$, change in taste $(2.4 \%)$, hydrocephalus (2.4\%), diplopia (1.2\%), and peritumoral edema (1.4\%). Headache was persistent at the last followup visit in $3.5 \%$ of cases at 10,10 , and 72 months postoperatively. Of the 2 cases of postoperative hydrocephalus, 1 was minimally symptomatic and has not required treatment more than 6 years after SRS and the other was managed with ventriculoperitoneal shunt placement and made a full recovery. The only instance of peritumoral edema was managed with a course of corticosteroids and resolved completely without long-term complications in the patient. There was no significant difference in lesion size or date of intervention when the cases were grouped by the presence or absence of any complication.

\section{Discussion}

For newly diagnosed VS, the management strategies available include SRS, MS, and observation. Observation is a viable option for lesions that are incidentally discovered or asymptomatic or for patients who are elderly or otherwise averse to treatment. MS and SRS offer unique advantages depending on the patient's demographic characteristics and the specific goals of therapy. The choice depends on the treatment goals of the patient and the experi- 

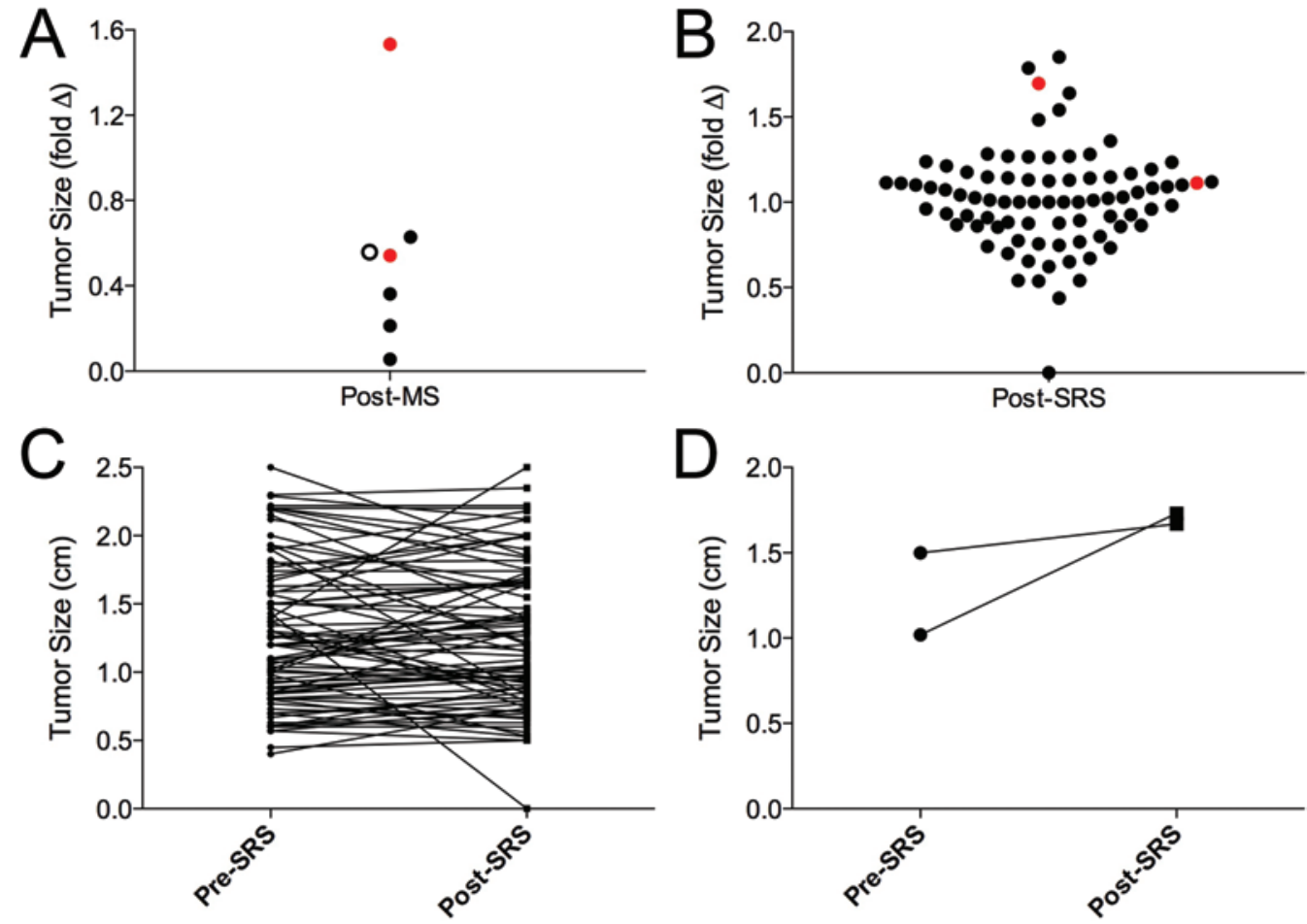

FIG. 4. Radiographic tumor control of the MS and SRS cases. A: The fold change in tumor size relative to preoperative imaging in the MS cases. The marker style indicates treatment failure (red circles), radiographic progression (open black circles), and radiographic stability (filled black circles). B: The fold change in tumor size relative to preoperative imaging in the SRS cases. The marker style indicates treatment failure (red circles) and treatment nonfailure (filled black circles). C: Absolute lesion size before and after SRS for all SRS cases. D: Absolute lesion size before and after SRS for SRS cases that failed treatment and required subsequent intervention. Figure is available in color online only.

ence of the treatment team. The focus of the present study was to compare outcomes between SRS and MS for VS in demographically matched patient groups. To the best of our knowledge, this is the largest reported matched cohort analysis to compare SRS and MS for VSs measuring less than $2.8 \mathrm{~cm}$.

Given the benign nature of VS, conservative management is appropriate for certain populations for which an intervention may be of more harm than benefit. ${ }^{6,8}$ This is particularly true for small, incidentally discovered, asymptomatic lesions in which a period of observation is often recommended as an initial step. When considering treatment options, it is essential for the physician and patient to discuss the natural history of the disease and define management priorities (hearing preservation, facial motor function, or cure). The tumor growth rate has been inversely correlated with hearing preservation, ${ }^{25}$ and it may be prudent to factor the tumor growth rate into the decision of when to pursue intervention. In general, intervention is warranted for larger lesions, for those demonstrating growth or symptom progression (hearing decline), or for patients who desire treatment after a discussion about the associated risks and benefits.

In our single-center, matched-cohort analysis of patients with treatment-naive unilateral VS $\leq 2.8 \mathrm{~cm}$, we found that both SRS and MS provide definitive tumor control. Notably, the SRS cohort had better hearing preservation and facial nerve function within the defined follow-up period. We also noted that hearing preservation was initially better within the SRS cohort, but that the difference in hearing preservation appears to lessen as the follow-up duration increases. Indeed, it was previously reported that the hearing loss associated with SRS is delayed, ${ }^{13}$ emphasizing the importance of the follow-up period. The median follow-up duration for SRS in our study was only 30.3 months, and it is likely that a longer follow-up duration would reveal a less significant difference in hearing preservation between the 2 treatment modalities. Our analysis demonstrated that by shifting the outcome from the preservation of serviceable hearing to the preservation of preoperative hearing status, which is a more sensitive outcome metric, the survival curves of hearing preservation for MS and SRS appeared to converge by 60 months. An update to this data set would therefore be warranted in 3 to 5 years in order to reassess hearing outcomes.

The current study examined only small- and mediumsized VS. Historically, SRS has been considered a viable treatment option only for small- and medium-sized tumors, but more recent series have demonstrated that SRS can provide adequate tumor growth control for larger lesions as well, ${ }^{20,29}$ although the control rate is not as good as that achieved with smaller tumors..$^{28}$ The concern in managing large VS using SRS arises from the fact that SRS can be associated with a small and transient expansion of the tumor before growth control or regression is observed. For large lesions, this transient expansion could exert a mass effect that compresses the adjacent brainstem structures. Similarly, there is concern that treatment failure 
TABLE 5. Most common complications by intervention

\begin{tabular}{lll}
\hline \multicolumn{1}{c}{ Variable } & \multicolumn{1}{c}{ MS } & \multicolumn{1}{c}{ SRS } \\
\hline Frequency (rate per procedure) & & \\
\hline Headache & $14(16.5)$ & $5(5.9)$ \\
\hline CSF leakage & $4(4.7)^{\star}$ & 0 \\
\hline Peritumoral edema & 0 & $1(1.2) \dagger$ \\
\hline Gustatory changes & $5(5.9)$ & $2(2.4)$ \\
\hline Hydrocephalus & $1(1.2) \ddagger$ & $2(2.4) \S$ \\
\hline Infection & $2(2.4) \uparrow$ & 0 \\
\hline Diplopia & 0 & $1(1.2)$ \\
\hline DVT & $1(1.2)^{\star *}$ & 0 \\
\hline LCN dysfunction & $1(1.2) \dagger \dagger$ & 0 \\
\hline Seroma & $1(1.2)$ & 0 \\
\hline
\end{tabular}

$\mathrm{LCN}=$ lower cranial nerve.

* None of these patients underwent treatment and all attained full recovery.

$\dagger$ This patient underwent treatment with corticosteroids and attained full recovery.

$\ddagger$ This patient underwent 2 high-volume spinal taps and attained full recovery.

$\S$ One of these patients did not undergo treatment and remained minimally symptomatic. The other patient underwent ventriculoperitoneal shunt placement and attained full recovery.

If One of these patients underwent debridement and the other underwent treatment with antibiotics. Both attained full recovery.

** This patient underwent anticoagulation therapy and attained full recovery. t† This patient did not undergo treatment and remained minimally symptomatic.

rates may rise as the tumor size increases. It has previously been reported that outcomes deteriorate and complications increase in parallel with tumor volume for both SRS and MS, so the need for a direct comparison study within this subgroup of tumors remains.

With respect to hearing preservation, SRS and MS each offer unique advantages and disadvantages. With hearingpreserving surgical approaches, preservation rates following MS are generally less than $50 \%$ but may be as high as $70 \%$ for properly selected cases..$^{19}$ For all MS cases, if hearing is preserved postoperatively, it can be considered "durable hearing preservation" as it is unlikely to change markedly in the absence of recurrent or progressive disease. In contrast, hearing preservation rates are higher immediately after SRS, but these rates declined over time to less than $25 \%$ at 10 years in one study. ${ }^{5}$ In our series, SRS demonstrated the superior preservation of serviceable hearing and preoperative hearing status over a median follow-up period of 30.3 months. It is likely that this difference would diminish as the follow-up duration for the SRS cohort increases beyond 60 months. More recently, there has been interest in the concept that hearing loss after SRS may depend on age, tumor margins, ${ }^{30}$ and cochlear dose..$^{15}$ Some believe that post-SRS hearing loss is a consequence of cochlear irradiation or the effect of continued adherence to the intact cochlear nerve. Based on current evidence, optimal hearing preservation can be achieved with a marginal dose of $\leq 12.5 \mathrm{~Gy}^{30}$ and a cochlear dose of $\leq$ $4.2 \mathrm{~Gy},{ }^{2,15}$ but it will be several years before studies report the long-term hearing preservation rates of this newer treatment paradigm. For patients who prioritize short-term hearing preservation and are cognizant of the risk of hear- ing loss over the next 10 years, SRS or observation might be preferable. Microsurgery is currently the only treatment option that offers the potential for long-term hearing preservation, but long-term data on more contemporary SRS dosing protocols are now accumulating.

The loss of facial nerve function is one of the most significant factors that affects postoperative quality of life, ${ }^{17}$ and it is most often iatrogenic rather than caused by the tumor itself. The rate of persistent postoperative facial nerve dysfunction for patients treated with MS can be as high as $30 \%$, depending on tumor size. ${ }^{1}$ In contrast, a recent systematic review found that SRS provides a low overall rate of facial nerve dysfunction, with modifiable variables such as patient age $\leq 60$ years, margin dose $\leq 13 \mathrm{~Gy}$, and tumor volume $\leq 1.5 \mathrm{~cm}^{3}$ prognosticating better outcomes. ${ }^{32}$ Consistent with prior reports, we found that SRS provided better preservation of facial nerve function compared with MS. While many patients focus on the symptoms they may have (such as hearing dysfunction, imbalance, or tinnitus), some patients focus less on the risk of new symptoms that they currently do not have at presentation (e.g., facial function). Yet, it is the opportunity to reduce the risk of facial dysfunction that is one of the most important advantages of radiosurgery.

For both hearing preservation and facial nerve function, the initial outcomes are better for SRS than MS. However, the hearing changes associated with SRS may be delayed by 5 or more years postoperatively; the median follow-up of the SRS cohort in our study was significantly shorter than 5 years, and it is possible that SRS and MS outcomes may converge over a longer follow-up period. As mentioned earlier, one of the major concerns in selecting SRS over MS is the risk that recurrent or progressive disease may jeopardize facial nerve and hearing outcomes in the long term after salvage intervention. ${ }^{9}$ The same is true with choosing MS first if a later recurrence is identified. Thus, it is particularly important to consider that, in our series, we found that disease progression as measured by the need for subsequent intervention did not differ between groups. This is important because for both hearing preservation and facial nerve function, one anticipated risk of pursuing SRS is that posed by delayed disease progression and the need for salvage surgery. If the disease progression rates are equivalent between MS and SRS, this concern for salvage surgery is moot and SRS and MS may be considered as equally efficacious therapeutic options for VS.

In our series, we report low overall complication rates for both SRS and MS. The most common complications were relatively benign, and the rate of permanent complications was very low for both treatment modalities. The most common benign complication for either treatment modality was headache. In most cases that presented with postoperative headache, the headache resolved within a relatively short period. The rate of persistent headache was equivalent for SRS and MS, which is interesting considering that the mechanism of postoperative headache in SRS has yet to be elucidated. Other potentially serious complications, such as DVT, hydrocephalus, and infection, were rare for both treatment modalities but less common with SRS. Within our series, no instances of mortality were reported, and none of the complications resulted in per- 
sistent disability in the patients treated with either MS or SRS. The only serious complication of SRS was 1 case of peritumoral edema that subsequently resolved. In contrast, there were several potentially serious complications of MS within our series, including 1 case of DVT, 4 cases of CSF leakage, and 1 case of infection. In all cases, early identification and aggressive management ensured that none of these potentially serious complications resulted in longterm disability for the patient.

In interpreting the results of our study, certain limitations should be noted. This is a retrospective, matchedcohort study designed to assess tumor control durability, hearing preservation, facial nerve outcomes, and complications following SRS and MS. Vestibular symptoms were not measured in a systematic or quantitative manner and thus could not be properly evaluated for analysis. A small report from our institution previously demonstrated that better vestibular outcomes can be obtained with the translabyrinthine approach. ${ }^{7}$ Future efforts will focus on the prospective collection of vestibular symptoms. While we were able to control for the effects of age and tumor size, it remains possible that the study populations may have differed in terms of unmeasured factors. The most notable shortfall of the current study was the relatively short follow-up duration for SRS, as this pertains to postoperative hearing function. In addition, many VS patients have decades of additional life expectancy, further emphasizing that the median follow-up duration in our study was relatively short. This may become an increasingly important factor as SRS is applied to younger patient populations. To better appreciate the significance of treatment modality on the quality of life of VS patients, longer follow-up and more direct assessments of quality of life would be desired. Finally, the study data were collected at 1 institution, and our outcomes are likely attributable to the experience of a center dedicated to VS treatment. The relative successes of various therapeutic modalities will likely vary with the experience of the surgeons, the techniques used, and the patients' treatment goals.

\section{Conclusions}

In summary, VS resection or radiosurgery for tumors $\leq$ $2.8 \mathrm{~cm}$ in diameter was associated with low overall morbidity, and the need for subsequent intervention was the same in both groups. SRS was associated with improved hearing and facial preservation rates and reduced morbidity, but with a shorter average follow-up interval. Facial function was excellent in both groups. As a result, for patients who are candidates for either treatment, the decision to pursue radiosurgery or resection should be based on the goals of the patient and the experience of the treating surgeons.

\section{References}

1. Arthurs BJ, Fairbanks RK, Demakas JJ, Lamoreaux WT, Giddings NA, Mackay AR, et al: A review of treatment modalities for vestibular schwannoma. Neurosurg Rev 34:265279, 2011

2. Baschnagel AM, Chen PY, Bojrab D, Pieper D, Kartush J, Didyuk O, et al: Hearing preservation in patients with vestibular schwannoma treated with Gamma Knife surgery. J Neurosurg 118:571-578, 2013
3. Benghiat H, Heyes G, Nightingale P, Hartley A, Tiffany M, Spooner D, et al: Linear accelerator stereotactic radiosurgery for vestibular schwannomas: a UK series. Clin Oncol (R Coll Radiol) 26:309-315, 2014

4. Benson K, Hartz AJ: A comparison of observational studies and randomized, controlled trials. N Engl J Med 342:18781886,2000

5. Carlson ML, Jacob JT, Pollock BE, Neff BA, Tombers NM, Driscoll CL, et al: Long-term hearing outcomes following stereotactic radiosurgery for vestibular schwannoma: patterns of hearing loss and variables influencing audiometric decline. J Neurosurg 118:579-587, 2013

6. Chung HT, Ma R, Toyota B, Clark B, Robar J, McKenzie M: Audiologic and treatment outcomes after linear acceleratorbased stereotactic irradiation for acoustic neuroma. Int J Radiat Oncol Biol Phys 59:1116-1121, 2004

7. Coelho DH, Roland JT Jr, Rush SA, Narayana A, St Clair E, Chung W, et al: Small vestibular schwannomas with no hearing: comparison of functional outcomes in stereotactic radiosurgery and microsurgery. Laryngoscope 118:1909-1916, 2008

8. Delbrouck C, Hassid S, Massager N, Choufani G, David $\mathrm{P}$, Devriendt D, et al: Preservation of hearing in vestibular schwannomas treated by radiosurgery using Leksell Gamma Knife: preliminary report of a prospective Belgian clinical study. Acta Otorhinolaryngol Belg 57:197-204, 2003

9. Friedman RA, Brackmann DE, Hitselberger WE, Schwartz MS, Iqbal Z, Berliner KI: Surgical salvage after failed irradiation for vestibular schwannoma. Laryngoscope 115:18271832,2005

10. Gait C, Frew EJ, Martin TP, Jowett S, Irving R: Conservative management, surgery and radiosurgery for treatment of vestibular schwannomas: a model-based approach to costeffectiveness. Clin Otolaryngol 39:22-31, 2014

11. Hajioff D, Raut VV, Walsh RM, Bath AP, Bance ML, Guha A, et al: Conservative management of vestibular schwannomas: third review of a 10-year prospective study. Clin Otolaryngol 33:255-259, 2008

12. Harris PA, Taylor R, Thielke R, Payne J, Gonzalez N, Conde JG: Research electronic data capture (REDCap) - a metadata-driven methodology and workflow process for providing translational research informatics support. J Biomed Inform 42:377-381, 2009

13. Hasegawa T, Kida Y, Kato T, Iizuka H, Yamamoto T: Factors associated with hearing preservation after Gamma Knife surgery for vestibular schwannomas in patients who retain serviceable hearing. J Neurosurg 115:1078-1086, 2011

14. Heman-Ackah SE, Cosetti MK, Gupta S, Golfinos JG, Roland JT Jr: Retrosigmoid approach to cerebellopontine angle tumor resection: surgical modifications. Laryngoscope 122:2519-2523, 2012

15. Kano H, Kondziolka D, Khan A, Flickinger JC, Lunsford LD: Predictors of hearing preservation after stereotactic radiosurgery for acoustic neuroma. J Neurosurg 111:863-873, 2009

16. Kondziolka D, Mousavi SH, Kano H, Flickinger JC, Lunsford LD: The newly diagnosed vestibular schwannoma: radiosurgery, resection, or observation? Neurosurg Focus 33(3):E8, 2012

17. Leong SC, Lesser TH: A national survey of facial paralysis on the quality of life of patients with acoustic neuroma. Otol Neurotol 36:503-509, 2015

18. Lin D, Hegarty JL, Fischbein NJ, Jackler RK: The prevalence of "incidental" acoustic neuroma. Arch Otolaryngol Head Neck Surg 131:241-244, 2005

19. Meyer TA, Canty PA, Wilkinson EP, Hansen MR, Rubinstein JT, Gantz BJ: Small acoustic neuromas: surgical outcomes versus observation or radiation. Otol Neurotol 27:380-392, 2006 
20. Milligan BD, Pollock BE, Foote RL, Link MJ: Long-term tumor control and cranial nerve outcomes following $\gamma$ knife surgery for larger-volume vestibular schwannomas. J Neurosurg 116:598-604, 2012

21. Myrseth E, Møller P, Pedersen PH, Lund-Johansen M: Vestibular schwannoma: surgery or gamma knife radiosurgery? A prospective, nonrandomized study. Neurosurgery 64:654-663, 2009

22. Myrseth E, Pedersen PH, Møller P, Lund-Johansen M: Treatment of vestibular schwannomas. Why, when and how? Acta Neurochir (Wien) 149:647-660, 2007

23. Pollock BE: Vestibular schwannoma management: an evidence-based comparison of stereotactic radiosurgery and microsurgical resection. Prog Neurol Surg 21:222-227, 2008

24. Stangerup SE, Tos M, Thomsen J, Caye-Thomasen P: True incidence of vestibular schwannoma? Neurosurgery 67:1335-1340, 2010

25. Sughrue ME, Kane AJ, Kaur R, Barry JJ, Rutkowski MJ, Pitts LH, et al: A prospective study of hearing preservation in untreated vestibular schwannomas. J Neurosurg 114:381385, 2011

26. Varughese JK, Breivik CN, Wentzel-Larsen T, Lund-Johansen M: Growth of untreated vestibular schwannoma: a prospective study. J Neurosurg 116:706-712, 2012

27. Whitmore RG, Urban C, Church E, Ruckenstein M, Stein SC, Lee JY: Decision analysis of treatment options for vestibular schwannoma. J Neurosurg 114:400-413, 2011

28. Williams BJ, Xu Z, Salvetti DJ, McNeill IT, Larner J, Sheehan JP: Gamma Knife surgery for large vestibular schwannomas: a single-center retrospective case-matched comparison assessing the effect of lesion size. J Neurosurg 119:463-471, 2013

29. Yang HC, Kano H, Awan NR, Lunsford LD, Niranjan A, Flickinger JC, et al: Gamma Knife radiosurgery for largervolume vestibular schwannomas. Clinical article. J Neurosurg 114:801-807, 2011

30. Yang I, Aranda D, Han SJ, Chennupati S, Sughrue ME, Cheung SW, et al: Hearing preservation after stereotactic radiosurgery for vestibular schwannoma: a systematic review. J Clin Neurosci 16:742-747, 2009

31. Yang I, Sughrue ME, Han SJ, Aranda D, Pitts LH, Cheung SW, et al: A comprehensive analysis of hearing preservation after radiosurgery for vestibular schwannoma. J Neurosurg 112:851-859, 2010

32. Yang I, Sughrue ME, Han SJ, Fang S, Aranda D, Cheung SW, et al: Facial nerve preservation after vestibular schwannoma Gamma Knife radiosurgery. J Neurooncol 93:41-48, 2009

\section{Disclosures}

The authors report no conflict of interest concerning the materials or methods used in this study or the findings specified in this paper.

\section{Author Contributions}

Conception and design: Golfinos, Hill, Choudhry, Kondziolka. Acquisition of data: Hill, Rokosh, Shinseki, Mansouri. Analysis and interpretation of data: Golfinos, Hill, Rokosh, Choudhry, Kondziolka. Drafting the article: Hill, Rokosh, Choudhry, Mansouri. Critically revising the article: all authors. Reviewed submitted version of manuscript: Golfinos, Hill, Rokosh, Choudhry, Shinseki, Friedmann, Roland, Kondziolka. Statistical analysis: Hill, Rokosh. Administrative/technical/material support: Golfinos, Hill, Roland, Kondziolka.

\section{Supplemental Information \\ Previous Presentations}

Portions of this work were presented in abstract form at the Annual Meeting of the American Association of Neurological Surgeons, Washington, DC, on May 2-6, 2015.

\section{Correspondence}

John G. Golfinos, Department of Neurosurgery, NYU Langone Medical Center, New York University, 530 First Ave., Ste. 8R, New York, NY 10016. email: john.golfinos@nyumc.org. 